\section{CONSIDERATIONS RESPECTING THE VARIOUS FORMS OF MALARIOUS AND MALARIOID DISEASE.}

By C. Handfield Jones, M.B., F.R.S., Physician to

\author{
St. Mary's Hospital.
}

No one can question that we gain much in every step we make towards fuller and clearer ideas of the nature of the great morbid actions with which, variously combined, we have constantly to deal. This knowledge, it seems to me, is what we want more than any other at the present time. Our diagnosis is not very often at fault; we can name a disease well enough, but we lack a full inspection into the nature of the process we observe, which alone could enable us to oppose it by a truly rational treatment. How much we need to know what it is that makes tuberculosis, rheumatism, pyrexia, inflammation, etc., so that we might be able, on recognising these morbid changes, to perceive with the mental eye the fundamental state from which they originated! The following review of one group of diseases may perchance aid some in obtaining a clearer view of their pathology, of the meaning of the various morbid phenomena they present, and of the mode of treatment that should be adopted in unusual and perplexing conditions.

It may be well to premise the following.

(1) The sympathetic plexuses are given off from special ganglionic centres, having communications chiefly of the nature of commissural connections with the cerebro-spinal system. The ultimate ramifications of the plexuses are almost wholly made up of the special sympathetic fibres, mingled with very few of the tubular kind. There can be no doubt that the sympathetic centres are capable of a distinct and independent action, apart from the cerebro-spinal.

(2) The sympathetic plexuses are distributed on the arteries of the abdominal organs, the thoracic, and the cranial.

(3) Paralysis of these plexuses dilates the arteries they accompany, and increases heat and tissue change in the locality where they are distributed; it establishes active hyperæmia, which in states of debility may pass into inflammation.

(4) The arteries of the rest of the body probably receive vaso-motor nerves from the cerebro-spinal which accompany them; the former are of the same structure as the sympathetic and it may be considered probable that a lesion of them would produce similar effects. I have found these vaso-motor nerves on careful microscopic examination, most distinctly, in the skin of the fingers and dorsum of the foot. There can scarcely be any doubt of their existence in other parts, but the search for them is very difficult on account of the subcutaneous fat and fibrous tissue.

(5) The action of malarious poison is sometimes paralysing, at others irritating. Neuralgic pain, rigors, or convulsions, must be regarded as signs of irritation; numbness, coma, or loss of muscular power, as signs of paralysing influence. These two effects may be produced at the same time in different nervous structures of the same body.

(6) It is probable that the nervous system is specially affected by malarious poison; $(a)$ because certain diseases of the head (otitis) produce paroxysms exactly resembling those of ague; $(b)$ because neuralgia is a common result of its action and paralysis not unfrequent; $(c)$ because means which arouse and invigorate the nervous system, such as cheerful excitement, ward off the disease, while all depressing emotions induce it; $(d)$ because quinine, the best remedy for malarious disorders, acts on the brain, producing in over doses deafness and sometimes convulsions; $*$ and opium, a drug of positive cerebral action, is also efficient in some conditions of malarious disease; $(e)$ because confirmed malarious disease especially prostrates and stupefies the nervous power, and because this is also often the effect of an incipient attack.

(7) The greater prevalence of malarious disease at some periods than at others seems to be ascribable to the medical constitution of the year rather than to a greater production of poison. If the tendency is to high (sthenic) inflammatory disorder, there will be little of ague and kindred affections; if the tendency is to cause a lowered state of vis nervosa, if nervous affections predominate, then malarious diseases, or those that resemble them, will be common also. It is probable that the

* In a case recently under my care it produced blindness and some deafuess. heat of tropical countries renders the endemic disorders so severe as they are, not only by its generating more and more powerful malarious poison, but by enfeebling the nervous system, diminishing its equilibrium and tone, and so rendering it more liable to bouleversement.

(8) There seems reason to think that other poisons besides the marsh miasm may act on the nervous system in a very similar manner, producing phenomena almost or quite identical with those of true malarious disease. The poison of influenza certainly seems capable of doing this.

(9) The type of disease which prevails at present appears to be very favourable to the occurrence of phenomena of nervedisorder, either of a prostrating or irritating kind, and of such disorders of the vascular system as are in great measure referable to a debilitated condition of its own special nerves.

The phenomena of the first stage of a complete ague paroxysm are depressed action of the heart, contracted and pale skin, neuralgic pains, irritation of motor nerves (rigors), arrest of secretions, short and laboured respiration, cerebral debility. The temperature of the blood is rising, and congestion is taking place in internal organs. The vitality of the various organs is partially prostrated, as by a shock, and this has no small share in promoting the occurrence of congestions.

This stage may be replaced by an attack of convulsions, or by a profound sleep from which the patient awakes in violent fever.

All the above phenomena may be regarded as the results of $a$ poisonous influence operating on the cerebro-spinal and sympathetic nervous systems. This acts eminently on the solar plexus, occasioning a sense of debility and distress about the epigastrium, paralyses the various plexuses which are offshoots from the solar, as well as others which are formed by the abdominal sympathetic, from which result dilatation of arteries, increased heat of blood, and local hyperæmia. At the same time the cerebro-spinal system is affected, in some parts debilitated, in others irritated. The hemispheres of the brain are evidently weakened and enfeebled in their actings. The sensory nerves are irritated, giving rise to neuralgic pains, and the motor, being similarly affected, produce the rigors. The shrinking of the skin is produced by the contraction of some organic muscular fibres in its corium, as well as by the occlusion of its arterioles. The arrest of the secretions shows that the vital power of the organs producing them is impaired, or in abeyance. The action of the heart is in most cases directly enfeebled, just as the contractile coat of the arteries is ; sometimes however, as described by Dr. Bell in cholera, and as I have seen in cardiac neuralgia associated with ague, it is rather enchained and embarrassed in its working by an abnormal, irritating stimulus. If death occurred during the latter state (which seems to be characterised by a distressing sense of cardiac oppression), I should expect to find the heart firmly contracted, as was observed in those dying of cholera by Virchow, and by Reinhardt and Leubuscher. The two latter observers mention that their examinations were made on those who died in the algide stage. The short and laboured respiration depends in part on congestion taking place in the lungs, but still more, I expect, on disordered innervation of the phrenic and other respiratory nerves. When the voluntary muscles and heart are deranged from their healthy action by disorder of their nerves as we have seen, it is only-to be expected that the respiratory muscles should suffer in a like way. In the case of the cold stage being replaced by an attack of convulsions, or one of coma, it may be supposed that the morbid influence has operated with uriusual force on the principal nervous centres devoted to voluntary motion in the first instance, and on the cortical substance of the hemispheres in the second.

The phenomena of the second stage are increased action of the heart, relaxation of the cutaneous arteries, paralysis of their vaso-motor nerves, the condition of other parts remaining about the same. The congestions commenced in the first stage are now apt to be increased as the blood stream is driven with force through relaxed arteries by a powerfully acting heart. Epistaxis, apoplectic effusion, intestiral hæmorrhage, rupture of the spleen, may thus be produced. The relaxation of the superficial arteries is only an extension of the condition of the rest of the arterial system, but the increased action of the heart is the reverse. What is its explanation? What cause shall we assign for the central organ of the circulation being in so different a state from all the more peripheral? It is to be remembered that the occurrence of reaction is a step in the direction of recovery, it indicates as is well known an effort of the vital powers against the depressing morbific cause. With reference to this, it may be suggested as probable, that the 
cause of the heart's acting vigorously, while the arterial system still remains relaxed and dilated, may lie in part in the difference of its contractile tissue, which certainly has a stronger and more vigorous life than the non-striated fibre of the arterial coats, and is therefore capable of recovering and resuming its activity sooner than they do. A still more efficient cause of the exaggerated action of the heart may be found, I think, in the elevated temperature of the blood, which acts upon it in the way of a stimulus. Exposure to moist heat, when cooling by evaporation is impussible, accelerates the pulse remarkably. M. James mentions that his own rose from 70 to 120 while he was immersed in an atmosphere charged with hot vapour, the temperature in the lower strata being $111 \cdot 4^{\circ}$, and in the upper $118^{\circ} 4^{\circ}$ Fahr. The same experimenter endured with little inconvenience a temperature of $170^{\circ} \mathrm{Fahr}$., when the air was dry. One cannot aver positively that the increased heat of the blood in the former instance caused the rapid movement of the heart, but it is certainly very probable that it was so. It is very intelligible that the heart would not ohey the stimulus of the hotter blood, until it began to recover from its primary state of enfeeblement and depression. On this view, we can see in reaction one of those beautiful compensating efforts which are often exhibited by nature in disease. The very agent which weakens and depresses the chief organ of the circulation, sets up a condition in the blood which counteracts the original mischief, or at least greatly tends to do so. In some persons, severe fatigue is followed by very marked pyrexia. This may be well accounted for on the view proposed. The expenditure of nervous force leaves the sympathetic in a semi-paralysed state; hence comes increased heat of blood, and from this again results increased cardiac movement.

The character and degree of reaction (speaking of it for a moment generally) will vary very much according to the nature of the poisonous influence, and the vital endowments of the system. In fevers of a low type, the muscular tissue of the heart may become so impaired in its quality that it answers but languidly to the stimulus of the hot blood. In the ardent inflammatory fever of tropical countries, especially when it affects new comers, the highly irritable contractile fibre responds energetically to the stimulus. Bloodletting is well borne in the latter, is injurious in the former.

Authors mention the occurrence of paroxysms consisting of a cold or a hot stage only. In the former case, it may be corsidered that the poison has operated chiefly on the cerebro-spinal system; no febrile reaction takes place, because the temperaature of the blood has not been increased by paralysis of the sympathetic. In the latter, the poison operates exclusively on the vaso-motor nerves, and therefore pyrexial phenomena alone are produced. That the poison should thus restrict its action in some cases to a certain department of the nervous srstem cannot be considered improbable, when we think how decidedly this is the case in various neuralgiæ.

Virchow attributes the reactionary excitement of the heart to paralysis of the vagus nerve; but, though this might very possibly increase the rapidity of its action, it would not, I conceive, augment its energy, which is certainly the most important feature of reaction. Moreover, careful experiments on the effects of dividing the vagi (Iieid's) do not show any very positive result in accelcrating the heart's contractions.

In the third stage, the tissues regain their power generally, secretions are poured out by the glands which have hitherto been inactive, the abnormal beat dimiuishes as the sympathetic nerves recover their tone, and the action of the heart becomes tranquil grailually.

In the alfide ferers, the prominent phenomena which we have to explain are the icy cold of the surface, the elevation of the temperature after death, the smallness or suppression of the pulse, the peculiar cerebral torpor, but without coma or loss of conscionsness, and the debility and depression. The same results of hyperæmic afflux and congestion in the cranial and abdominal viscera are found as in the other forms of ague fever. The skin is not always pale, but sometimes of natural colour, sometimes livid, or covered with livid spots. One may read these cases as the results of a morbid influence, which operates more after an irritating than a paralysing manner. The brain is evidently rreatly enfeebled, but rather, it would seem, in the same way as a sensory nerve, when it becomes numbed during an attack of neuralgia, a condition which Romberg denominates anæsthesia dolorosa. This cerebral numbing appears more like the result of a peculiar irritation, than simple loss of power. It is carried to a still greater degree in certain soporose fevers, where it produces absolute coma, which may be relieved by opium. A similar state prevails in the vaso-motor nerves of the limbs and surface; they are irritated and excited, instead of being paralysed, as during pyrexia. It is reasonable to suppose that the icy cold may, at least in part, be the result of this state; as it was found in Bernard's experiments that, when the upper end of the divided sympathetic was galvanised, the temperature fell below the normal figure. The elevation of the temperature after death seems necessarily to imply that some cause was acting during life to depress the heat that should have been normally developed. This cause is probably the irritated state of the vaso-motor nerves. The livid colour of the surface, like the leaden hue of cholera, is to be regarded, I believe, as the result of tissue paralysis; in consequence of which, and of arterial occlusion, stagnation occurs in the capillaries and small veins. A remarkable case recently fell under my notice, in which the nose and hands became cold and of a dull livid red on exposure to the open air, even in the middle of June. The other symptoms were extreme nerve-debility, tremors, and sweats.

It does not appear to me possible to account for such phenomena solely on the ground of arterial contraction. I may quote the following observations from my note-book, in further illustration of this state: "Frog's web, arteries of the part rather contracted, the blood in numerous capillaries stagnant, filling them up with an uniform red mass; the application of warmth dilates the arteries, and induces free flow in the capillaries." Wharton Jones records similar observations. This condition, which has some analogy to that existing in some algide fevers and in cholera, is widely different from that which is commonly observed as the result of cold operating upon a quite healthy part, which it renders exsanguine and pale. 'To quote again: "The effect of warmth and cold upon the circulation is very striking; by cooling the web with cold water, some arteries are brought to be completely contracted and empty, and the transit of red and white globules through the capillaries ceases, so that they appear empty; but as soon as the web is dried and warmed, the artery is found expanded and transmitting a full current, and the capillaries at the same time become filled with red corpuscles." It seems clear that, in these instances, the vital condition of the tissue alone makes all the difference, whether the capillaries are filled with corpuscles or not. If the tissue be quite healthy, the capillaries, during arterial occlusion by cold or other causes, will be traversed orily by plasma, and will be empty, or nearly so; if the vital power of the tissue be weakened, the blood congests and fills up the capillaries, but does not adhere to their walls, and the congestion is dissipated by free flow ; if the nutrition foree be perverted, as in sthenic intlammation, creating local attraction of a morbid kind between the blood and tissue, then stasis occurs, which is not removed, but only aggravated, by dilatation of the arteries and free blood flow. The existence of arterial contraction, amounting sometimes to occlusion, would account for the smallness or obliteration of the pulse, supposing it to extend to the radial and arteries of the same size.

The state of the heart probally varies, being in some cases directly enfeebled, in others enchained or embarrassed in its acting by morbid stimulus. Its movement does not seem to be accelerated into reactionary excitement, or only partially, even during the decline of the paroxysm. This is to be accounted for, partly by the cerebro-spinal system being more affected than the sympathetic, partly by the depressing influence being too intense to be overcome by the somewhat heated blood. It seems reasonable to think that in cases of this kind, on the view that hyperirritation of the vaso-motor nerves was chiefly concerned in causing the peculiar symptoms, the administration of opium with some diffusible stimulus would be beneficial.

In the comatose fevers, the coma may result from the palsying or enchaining action of the malarious poison telling upon the cerebral hemispheres, in which case it will occur in the earlier period of the attack, and will be relieved by opium. Or it may proceed from increased flow of blood to the brain, and excessive congestion tending to effusion, when it will occur in the reaction period, and will be relieved by biood-letting, local and general, cola to the head, and quinine.

In syncopic fevers (terciera syncopal of Pera), the heart is paralysed more or less completely ly the malarious influence acting on its nerves, just as a voluntary muscle, or set of muscles, may be. The syncope occurs, as far as I am aware, at the commencement of the cold stage. It may be difficult to distinguish these cases from others approximating more to the algide group, in which there is complete suppression of the pulse; but the heart, so far from being paralysed, is heard on auscultation acting violently, as if striving to overcome some obstacle, which is probably the occluded state of the arteries. 
The character of the impulse and sounds of the heart will generally enable us to make a diagnosis. The distinction is of great moment; for in the first case stimulants would of course be necessary, in the second sedative measures.

Local palsies of any motor or sensory nerve, or local neuralgiæ or convulsions, may occur, the affected nerves being either irritated or enfeebled.

If jaundice, co-existing with free bile flow, or any other secretion flux occur, it indicates that the vaso-motor nerves of the part are palsied, whence comes dilatation of blood-vessels, byperæmia, and over-activity of the gland tissue.

The formation of a splenic tumour results from excessive flow of blood to the part, and increased formation of splenic tissue, i.e. increased nutrition. It is therefore quite analogous to a gland flux. Both occurrences illustrate well the develop. ment of increased activity in the affected tissue as the result of paralysis of its vaso-motor nerves.

The common occurrence of dysentery, either primarily from exposure to malaria, or as a sequel or complication of malarious fever, is evidently a localisation of the morbid action, or it may be a concentration. The vaso-motor nerves of the large intestine are paralysed, and the usual results take place. It is to be mentioned, that the condition of a part, as to vital power, has much to do in determining the occurrence of inflammation after hyperæmia has been set up in the way supposed. If the vital power be tolerably sound, the part may remain unaltered, or may exhibit greater activity, as in the case of the gland flux; but, if the vital power be depressed, severe inflammation may result, e.g. purulent ophthalmia after section of the sympathetic in the neck. Though we can by no means see thoroughly into the matter, yet it does appear that there is a relation between the tissue power, the functional energy of a part, and its blood supply. In the healthy working of the apparatus, the former takes the initiative; and the byperæmic afflux is subordinate, and ministers to the calls of the tissue in its increased nutrition. In inflammatory disorder, the vital power, which can use the blood for normal purposes, is depressed, and the hyperæmic aftlux predominates, issuing in wasteful effusions of corpusculating fluids, mucus, or pus. Nay, it seems highly probable that in conditions of depressed vitality, as in frostbite the scorbutic dysentery of the Crimea and elsewhere, the existence of hyperæmia, even in moderate amount, acts as a morbid and destructive stimulus to the weakened part.

Having thus endeavoured to view some of the multifold manifestations of malarious disorder in the light of recent neuropathological research, it may be well to consider shortly what bearing these views have on other kinds of fever. It will be sufficient to take for examination two types, to one or other of which all approximate; viz., first, the severe inflammatory fever of tropical countries; second, the ordinary low fever, typhus, or typhoid, of our own. The phenomena of the first are, as described by Dr. Copland, slight chills only at the onset, subsequently "strong, full, hard, accelerated pulse", great heat, healache, and sometimes violent delirium, or maniacal excitement. Free venesection (to three or six pounds) is necessary. If in such fevers, on the above view, we admit the pyrexial condition to depend essentially on paralysis of the sympathetic, still it seems absolutely necessary to assume that the other tissues are in a state differing greatly from that which they manifest in many malarious, and all low fevers. The poison which has paralysed the sympathetic, has in no degree weakened the heart or the other organs. Hence the heated blood, acting on structures vigorous and prompt to respond to the stimulus, excites reactionary phenomena of great violence. It is the task of the physician to moderate these, as, if allowed to run their course unchecked, they induce a period of dangerous or fatal exhaustion.

How very apposite to this is all that we observe in the second type-the low fevers! Here every part alike bears the stamp of depression and debility. The sympathetic system is palsied, and hence the febrile heat, and the proneness to hyperæmia in various parts. The heart is enfeebled, and often needs stimulus to keep its action from failing; its very fibre often tends to soften and degenerate. The brain is enfeebled, and hence the low delirious wandering or the sopor. The voluntary muscles declare their atony by the down-sunk posture of the patient.

The lesson of this seems to be, that while we incline, as we may reasonably do, towards the theory of the nerve origin of pyrexial phenomena, we must not lose sight of the fact that the other corporeal systems are also affected to a greater or less degree by the fever poison, and that the event of the disease may in great measure depend on the amount of vital resistance and energy which they retain. In malarious fevers the nervous system (cerebro-spinal and sympathetic) is certainly far more affected than the others. In low fevers all the systems alike seem to be paralysed and depressed. In sthenic fevers no system, except the sympathetic, seems to have suffered depression, but perhaps to be rather in a state of abnormal irritability. Thus one physician may choose for his motto, " $\mathrm{He}$ fed fevers"; another, "He toned or soothed fevers"; a third, "He bled fevers"; each according to the kind he had most to deal with.

\section{DEFICIENCY OF FINGERS TRANSMITTED THROUGH SIX GENERATIONS.}

By Draper Mackinder, M.D., Gainsborough.

UNDER the title of "Hereditary Formations", a letter from $\mathrm{Mr}$. Willis, of Plumstead, appeared in the Lancet for Sep. 5th, wherein the writer mentions a repetition of an abnormal parental type through six generations. Believing with Mr. Willis that so decided a manifestation of a peculiarity in the progenitor is rarely extended through so many links in the descending family chain, I beg to forward, for the edification of the curious in such matters, an account of an equally interesting case, where there has been a deficiency instead of a redundancy of parts through several generations.

A Mrs. Thomas Chapman, of this town, on the 31st of March last, was attended in her confinement by my assistant, Mr. Capron. In consequence of an absence of certain useful members of the body, to be particularised hereafter, the mother was very naturally anxious to know if her babe had been modelled more in accordance with the Paradisian prototype than with her imperfectly sculptured self; and we can well imagine the parental joy on hearing that, to non-professional observers, her firstborn daughter would readily pass muster as a fair specimen of the daughters of England.

In the presence of Mr. Capron's successor, Mr. Smallman, and three generations of the family in question, I obtained the following particulars, which I will endearour to give in an intelligible form, tracing the generations backwards.

Generation I. The Infant. The middle phalanges of the eight fingers are wanting, the last phalanges articulating with the first, and giving a peculiar short and tapering appearance. The nails of both little fingers are absent, and that of the right ring finger is abortive. The toes are all nearly of the same length, and well suited for the square-toed boots.

Generation II. The Mother. The middle phalanges of eight fingers and all the nails are absent. The first phalanges articulate with the last, which are much shorter than common, and have the exact appearance of having undergone amputation at the middle of each third phalanx by a transverse flap. The fourth toe of each foot has only the first phalanx.

Mrs. Chapman is the youngest of a family of nine, the seven elder of whom and their children were of legitimate conformation.

Her brother, the eighth child, who was drowned at sea, had the ring and little fingers of both hands minus the second and third phalanges and nails; and the fourth and fifth toes of each foot were united by a web.

Generation III. The Grandfather possessed only the first phalanges of the eight fingers, the thumbs being flattened, and the phalangeal articulations very imperfect.

He had two brothers and ten sisters, of whom

One sister had the eight middle phalanges and the nails wanting.

A second sister had the third middle phalanges and nails wanting.

Another sister had the right index normal, and seven fingers minus the second and third phalanges and nails.

A brother had the same malformations as the last mentioned sister.

All the four had an irregularity about the toes. The other brother and sister were all riolht.

One of his brothers married, and had four daughters. The first was properly formed; the other three wanted the second phalanges and nails to all the fingers. Of the families of the other brother and his sisters nothing accurately is known.

Generation Iv. The Grandfather's Mother and three of his sisters, like himself, were all mimus the second and third phalanges and nails of eight fingers, and the second phalanges of the fourth toes.

Generation v. His Maternal Grandmother, and 